\title{
Gender Based Compositional Fluctuations in Gut Microbiota in Hypertension
}

\author{
Mushtaq $\mathbf{N}^{1}$, Hussain $\mathbf{S}^{2}$, Yuan $\mathrm{L}^{1}$, Zhang $\mathbf{S}^{1}$, Li $\mathbf{H}^{1}$, Ullah $\mathbf{S}^{1}$ and Xu $\mathbf{J}^{\mathbf{1}^{*}}$
}

${ }^{1}$ Department of Pathogenic Microbiology and Immunology, School of Basic Medical Sciences, Xi'an Jiaotong University, 710061 Xi'an, China

${ }^{2}$ Department of Molecular Biology and Biochemistry, School of Basic Medical Sciences, Xi'an Jiaotong University, 710061 Xi'an, China

\begin{abstract}
Background: Emerging evidence suggests that human gut microbiota plays critical role in maintaining physiological homeostasis, and gut dysbiosis is involved directly or indirectly in cardiovascular diseases including hypertension.
\end{abstract}

Aim: The aim of present study was to investigate whether there were sex based compositional differences in gut microbiota of patients with hypertension.

Methods: Fecal samples were collected from male and female hypertensive patients, and healthy individuals from Xi'an, Shaanxi province of China. Touchdown PCR and denaturing gradient gel electrophoresis (PCR-DGGE) with primers specifically targeting $\mathrm{V} 3$ region of $16 \mathrm{~S}$ rRNA, and real time quantitative PCR (qPCR) were performed to characterize all the samples. High-throughput sequencing of the V3-V4 regions was performed on Illumina HiSeq 2500.

Results: Diversity and richness indices for the gut microbiome were compared among the four groups. The relative abundance of Firmicutes was found increased in hypertensive groups (both female and male patients) while that of Bacteroidetes was decreased in male hypertensive patients only, as compared to the healthy male subjects. The most differentially abundant bacterial taxa belonged to the genera Prevotella and Megasphaera in female hypertensive patients and Megamonas in male hypertensive patients. The female and male hypertensive patients also showed different dominant phylotypes.

Conclusion: These results demonstrated that there were sex based differences in gut microbial composition of patients with hypertension. Our findings indicate Prevotella, Megasphaera and Megamonas as the potential sexspecific biomarkers of hypertension, and need to be further evaluated.

Keywords: Hypertension; Gut microbiota; DGGE; Real Time qPCR; High-throughput sequencing

\section{Introduction}

Hypertension (HTN) or high blood pressure nowadays is emerging as a threatening public health issues as it is a modifiable risk factor for diseases like stroke and cardiovascular anomalies $[1,2]$. Efforts to lower down the blood pressure (BP) become fruitless in elderly people with the habits of taking unhealthy diet, excessive use of alcohol, less physical activity, obesity and depression [3-6]. Strategic management and training is needed to overcome hypertension related mortality [7]. It may differ in lieu of age, sex and other factors [6,7-9]. It is observed that both in humans and animals, gender based differences in hypertension involve biological and behavioral factors [10]. Overall, hypertension is less prevalent in females than males, and the biological factors including sex hormones and other chromosomal and biological sex differences safeguard women against hypertension. These differences vividly become prominent during adolescence and continue to the adulthood until the women reach menopause. At this stage these gender based differences become almost insignificant $[10,11]$. It has been observed that gut microbiota regulate sex hormones mediated autoimmunity more effectively in women than men [12]. These sex hormones are known to play critical role in the development of cardiovascular diseases $[13,14]$. Other studies have highlighted the association of intestinal microbiota with metabolism of phosphatidylcholine and cardiovascular risk [15].

Earlier, a couple of reports proposed that gut microbiota may be involved in the maintenance of blood pressure homeostasis and that a compositional fluctuation in the gut microbiome may probably be implicated to the increased risk of developing hypertension [1618]. In addition, hypertension is associated with increased risk of arterial thrombotic disease. Among other factors, enhanced platelet activity contributes significantly to this phenomenon [19]. Recently, it was reported that gut microbioa directly contribute to platelet hyperreactivity and enhanced thrombosis potential in carotid artery thrombosis animal models (In vivo) [20]. Besides, the compositional fluctuation in the individuals' gut microbial community is linked with the environmental as well as host genetic factors [21,22]. Certainly, diet is considered as one of the major factors that can affect gut microbiota composition, individually and chronologically [23,24]. Sex is another important factor and there is no other view point that it has a significant impact on physiology and behavior [25], however there are controversial reports on sex based differences in the gut microbial composition. Some studies suggest that gender has no or very limited effect on gut microbiota [26-28], while others emphasize that there

${ }^{*}$ Corresponding author: Jiru $\mathrm{Xu}$, Department of Pathogenic Microbiology and Immunology, School of Basic Medical Sciences, Xi'an Jiaotong University, Yanta West Road No. 76, 710061 Xi'an, Shaanxi Province, China, E-mail: xujiru@mail.xjtu.edu.cn

Received October 01, 2018; Accepted October 23, 2018; Published October 30 , 2018

Citation: Mushtaq N, Hussain S, Yuan L, Zhang S, Li H, et al. (2018) Gender Based Compositional Fluctuations in Gut Microbiota in Hypertension. Cardiovasc Pharm Open Access 7: 248. doi: 10.4172/2329-6607.1000248

Copyright: (c) 2018 Mushtaq N, et al. This is an open-access article distributed under the terms of the Creative Commons Attribution License, which permits unrestricted use, distribution, and reproduction in any medium, provided the original author and source are credited. 
are significant differences in microbiota arrangement between sexes [29-31], and the commensal microbial population may influence sex hormone levels [12,32].

Recently, we have reported the gut microbiota compositional analysis and the efficacy of 5-fluorouracil in colorectal cancer rat model [33]. Currently we are working on 'role of gut microbiota in the development and management of hypertension, and related diseases', evaluating multiple factors including sex. In the present study, we have analyzed the gut microbial community of the hypertensive patients between the two sexes comparing with their healthy counterparts and have identified sex-specific microbial biomarkers for the diagnosis of hypertension. The current study uses the modern metagenomic techniques including High-throughput sequencing and Real-Time qPCR to evaluate the gender based gut microbial diversity and similarity in the human hypertensive patients along with the healthy controls.

\section{Materials and Methods}

\section{Study participants and design}

This study included 30 male hypertensive patients (MHT) and 25 female hypertensive patients (FHT), ages ranging between $40 \mathrm{y}$ and 75 y. 30 healthy volunteers included 15 males (Male Control GroupMCG) and 15 females (Female Control Group-FCG), aged between 40 $\mathrm{y}$ and $70 \mathrm{y}$. A full written consent from all the participants was taken and a questionnaire regarding gender, age, body weight and health status of the study participants was filled at the time of sampling. BP of the individuals was noted with a digital sphygmomanometer and three consecutive values were taken with a 5 min gap. Fecal samples were collected in sterile cups and were stored immediately at $-80^{\circ} \mathrm{C}$ until the DNA extraction was carried out. None of the patients and healthy individuals had diabetes, obesity and any record of chronic metabolic diseases. In addition, the participants had no history of antibiotics, probiotics and prebiotics usage from last 30 days, at the time of sampling.

\section{Ethical statement}

The current study was performed under the guidelines of World Medical Association and Declaration of Helsinki. The sampling and experimental procedures were performed with the approval of Ethical Committee of the Xi'an Jiaotong University, School of Medical Sciences and an informed written consent was obtained from all of the participants including male and female hypertensive patients as well as healthy volunteers.

\section{DNA extraction}

After thawing the fecal sample, bacterial DNA was extracted using the Qiagen QIAamp MiniStool Kit (QIAGEN, Hilden, Germany), according to the manufacturer's instructions and the extracted DNA concentration was estimated by absorbance at $260 \mathrm{~nm}$ (A260) with a Nanodrop spectrophotometer (Nanodrop Technologies, Wilmington, DE, USA).

\section{Analysis of gut microbiota by PCR-DGGE}

Universal primers for $\mathrm{V} 3$ region of $16 \mathrm{~S}$ rRNA gene were used to amplify the intestinal microbiota of the study samples by using total fecal DNA as a template for PCR-DGGE analysis. Each $50 \mu$ PCR reaction mixture contained $1 \mu \mathrm{l}$ of each primer (Table 1 ), $1 \mu$ l of deoxynucleotide triphosphate (dNTPs) mix (10 mM), $5 \mu \mathrm{l}$ of $\mathrm{MgCl}_{2}(25 \mathrm{mM}), 5 \mu \mathrm{l}$ of $10 \mathrm{x}$ buffer, $0.4 \mu \mathrm{l}$ of Taq DNA polymerase (TaKaRa, Japan), and $2 \mu \mathrm{l}$ of total fecal DNA. PCR amplification was performed in an automated thermocycler (ABI2720, USA) using touchdown PCR program for increasing the specificity of PCR reactions. The PCR thermal profile conditions include; initial denaturation at $95^{\circ} \mathrm{C}$ for $5 \mathrm{~min}$, followed by denaturation at $95^{\circ} \mathrm{C}$ for $1 \mathrm{~min}$, annealing at $65^{\circ} \mathrm{C}$ for $1 \mathrm{~min}$ and extension at $72^{\circ} \mathrm{C}$ for $1 \mathrm{~min}$. The annealing temperature was decreased by $1^{\circ} \mathrm{C}$ every second cycle until a touchdown $55^{\circ} \mathrm{C}$ reached, at which 10 additional cycles were carried out, followed by final extension at $72^{\circ} \mathrm{C}$ for $10 \mathrm{~min}$ [34]. Post-PCR gel electrophoresis was performed to confirm the amplicons. Negative controls included all the components of the reaction mix except the DNA template was run along with each reaction.

After PCR amplification, $15 \mu$ of each PCR product was mixed with $6 \mu \mathrm{l}$ of loading dye. Denaturing gradient gel electrophoresis (DGGE) was performed using the DCode ${ }^{\mathrm{TM}}$ Universal Mutation Detection System (Bio-Rad, Hercules, CA, USA) on $16 \mathrm{~cm} \times 10 \mathrm{~cm} \times 1 \mathrm{~mm}$ gels. The sequence-specific separation of the amplicons was obtained with $10 \%(\mathrm{w} / \mathrm{v})$ polyacrylamide (acrylamide-bis, $37.5: 1)$ gel in $1 \times$ Tris-acetate EDTA (TAE) buffer, containing 65-35\% linear denaturant gradient. The $100 \%$ denaturing solution contained $7.0 \mathrm{M}$ urea and $40 \%$ (vol/vol) deionized formamide. Electrophoresis was performed at a constant voltage of $90 \mathrm{~V}$ at $60^{\circ} \mathrm{C}$ for $13 \mathrm{~h}$. After completion of the run, the gel was stained with $5 \mu \mathrm{g} / \mathrm{ml}$ ethidium bromide solution for $30 \mathrm{~min}$, washed with deionized water and viewed under BIO-RAD Gel Doc 2000 system. DNA Marker (DL2000) was used as standard reference to compare the bands in the gel. Quantity One software (Bio-Rad, USA) was used to evaluate the bacterial diversity of the study groups by analyzing the number of bands and the bands intensities of DGGE profiles. The diversity of bacterial taxa was determined by the ShannonWeaver index $\left(\mathrm{H}^{\prime}\right)$. The similarity score and cluster analysis of DGGE profiles were determined by the UPGMA method based on the Dice similarity coefficient (band-based). Nonparametric statistical analysis

\begin{tabular}{|c|c|c|c|c|c|}
\hline Groups & Target Gene & Primer & Sequence (5'-3') & Product Size (bp) & Ref. \\
\hline \multirow{2}{*}{ PCR-DGGE } & \multirow{2}{*}{$\begin{array}{c}\text { Bacteria } 16 \text { s rRNA gene V3 } \\
\text { region }\end{array}$} & $341 \mathrm{~F}^{\mathrm{a}}$ & CCTACGGGAGGCAGCAG & \multirow{2}{*}{193} & \multirow{2}{*}{ [34] } \\
\hline & & $534 \mathrm{R}$ & ATT ACCGCGGCTGCTGG & & \\
\hline \multirow{8}{*}{$\begin{array}{l}\text { Real } \\
\text { time } \\
\text { PCR }\end{array}$} & \multirow{2}{*}{ Bacteroides } & Bac F & AAGGGAGCGTAGATGGATGTTTA & \multirow{2}{*}{193} & \multirow{2}{*}{ [57] } \\
\hline & & Bac $R$ & CGAGCCTCAATGTCAGTTGC & & \\
\hline & \multirow{2}{*}{ Prevotella } & Pre $F$ & ACAGTAAACGATGGATGCC & \multirow{2}{*}{513} & \multirow{2}{*}{ [58] } \\
\hline & & Pre $\mathrm{R}$ & GGTCGGGTTGCAGACC & & \\
\hline & \multirow{2}{*}{ Clostridium } & Clos F & CGGTACCTGACTAAGAAGC & \multirow{2}{*}{429} & \multirow{2}{*}{ [59] } \\
\hline & & Clos $\mathrm{R}$ & AGTTTGATTCTTGCGAACG & & \\
\hline & \multirow{2}{*}{ Escherichia coli } & E.coli F & CATTGACGTTACCGCAGAAGAAGC & \multirow{2}{*}{190} & \multirow{2}{*}{ [59] } \\
\hline & & E.coli R & СTCTACGAGACTCAAGCTTGC & & \\
\hline
\end{tabular}

aA 5' GC-clamp (CGC CCG CCG CGC GCG GCG GGC GGG GCG GGG GCA CGG GGG G) was added for DGGE analysis.

Table 1: Primers deployed in PCR-DGGE and Real-time PCR. 
was performed using IBM SPSS Statistics (v20) software. Results are expressed as mean values and standard deviations.

\section{Sequencing of selected bands from DGGE gels}

The distinct, prominent and common bands were cut from the gel with a sterile razor, washed with RNAse Free water, re-suspended in $20 \mu \mathrm{l}$ of the RNAse Free water and stored at $4^{\circ} \mathrm{C}$ overnight for the diffusion of DNA. PCR was performed again with the diffused DNA using V3 region primers $341 \mathrm{~F} / 534 \mathrm{R}$ this time without GC clamp (initial denaturation at $95^{\circ} \mathrm{C}$ for $5 \mathrm{~min}$, followed by denaturation at $95^{\circ} \mathrm{C}$ for $1 \mathrm{~min}$, annealing at $65^{\circ} \mathrm{C}$ for $1 \mathrm{~min}$ and extension at $72^{\circ} \mathrm{C}$ for $1 \mathrm{~min}$.). Re-amplified PCR products were sequenced by Shanghai Sangon Biological Engineering Technology Service Co. Ltd. (Shanghai, China). Sequences were analyzed with Chromas v2.23 (Technelysium, Tewantin, Australia) and their similarity was confirmed by using BLAST and Seqmatch softwares.

\section{Real time quantitative PCR analysis}

Real-time PCR quantification was performed using the StepOne v2.3 (ABI, USA) system. Real-time PCR primers are depicted in Table 1. To calculate the absolute copy number of the Bacteroides spp., Prevotella spp., Clostridium spp., and Escherichia coli within the test samples, QuantiFast SYBR Green PCR kit (Qiagen) was used and a standard curve was prepared from a serially diluted plasmid DNA (of known concentration) ranging from $10^{2}$ to $10^{8}$ copies/g of feces to quantify the test samples. The resultant bacterial populations were expressed as $\log 10$ bacterial replica counts in 1 gram of the fecal mass. Statistical analysis was performed by using Student's $t$ - test.

\section{High-throughput sequencing and data analysis}

30 sampleswererandomlyselectedformetagenomichigh-throughput sequencing analysis. 515-F (GTGCCAGCMGCCGCGGTAA) 806-R (GGACTACHVGGGTWTCTAAT) primers were used to develope the amplicon libraries [35]. The libraries were sequenced on an Illumina HiSeq 2500 platform according to the manufacturer's instructions. The Raw data obtained, were screened and assembled by QIIME [36] and FLASH [37] software packages. The UCLUST method [38] was used to cluster the sequences into Operational Taxonomic Units (OTUs) at an identity threshold of $97 \%$. Meanwhile, the RDP classifier [39] was used to assign each OTU to a taxonomic level. Alpha Diversity analysis, including Shannon and Simpson diversity index, Chao 1, ACE and Good's coverage, was performed with QIIME (Version 1.7.0). Student's $t$-test was used to calculate the significant differences in alpha diversity between the groups. The relative abundance of phyla, family, genera and species in each sample was calculated and compared between the study groups. All statistical analysis was carried out by IBM SPSS (v20) software.

\section{Results}

\section{Biochemical measurements of the study participants}

Other than systolic and diastolic blood pressure, female patients had higher fasting blood glucose $(P=0.02)$ and triglycerides $(P=0.01)$ levels. On the other hand, male hypertensive patients showed increased levels of fasting blood glucose $(P=0.02)$, triglycerides $(P=0.03)$ as well as low-density lipoproteins $(P=0.04)$ as compared to their healthy groups (Table 2).

\section{Sequencing and statistical analysis of DGGE profile}

Dominant bands from different positions within the DGGE profiles were further analyzed through sequencing. Bacteroidetes and Firmicutes were appeared as the dominant bacterial phyla within the study groups; however, the percentage of different genera was different among the four groups (Table S1). The detailed results from DGGE analysis are shown in (Figures 1A and 2A). The DGGE profiles band number and the Shannon diversity index $\left(\mathrm{H}^{\prime}\right)$ analysis, in order to estimate the gut microbial diversity within the study groups is summarized in Table 3. The resultant distinguishable findings elucidate that gut microbial diversity showed no significant difference in the hypertensive groups as compared to their healthy counterparts. Furthermore, Dice similarity coefficient and UPGMA, to determine the extent of similarity of all the DGGE profiles, were applied (Figures $1 \mathrm{~B}$ and $2 \mathrm{~B}$ ). The assessed values of the similarity coefficient of female hypertensive patients and healthy controls with mean index values were $44.78 \pm 12.46$ and $41.78 \pm 12.91$, respectively. The similarity coefficient of male hypertensive patients and healthy control with mean index values was $42.69 \pm 11.29$ and $40.32 \pm$ 8.52 , respectively (Table 3 ). The compiled statistical data of the samples from hypertensive and control groups analyzed by Dice coefficient showed lesser values among the groups, that in turn affirms that gut microbiota of hypertensive patients (male and female) varies from the healthy controls (male and female) respectively.

\section{Real-time PCR}

Real-time PCR analysis was performed and evaluation of the mean index value demonstrated that the copy number of Bacteroides spp. and Clostridium spp. were significantly decreased and increased, respectively in the male hypertensive patients as compared to the male healthy controls $(P<0.05)$. While the Prevotella spp. was significantly

\begin{tabular}{|c|c|c|c|c|c|c|}
\hline & \multicolumn{2}{|c|}{ Female } & \multirow{2}{*}{ P-value } & \multicolumn{2}{|c|}{ Male } & \multirow{2}{*}{ P-value } \\
\hline & HTN $(n=25)$ & CG $(n=15)$ & & HTN $(n=30)$ & CG $(n=15)$ & \\
\hline Age (yrs) & $64.21 \pm 11.52$ & $58.85 \pm 12.42$ & 0.47 & $65.1 \pm 11.4$ & $61.93 \pm 9.77$ & 0.75 \\
\hline Weight (kg) & $55.5 \pm 5.86$ & $62.7 \pm 5.02$ & 0.63 & $63.61 \pm 4.35$ & $67.56 \pm 5.98$ & 0.18 \\
\hline SBP, mmHg* & $160.15 \pm 16.63$ & $120 \pm 9.23$ & $<0.0001$ & $175.45 \pm 9.23$ & $118.13 \pm 6$ & $<0.0001$ \\
\hline DBP, $\mathrm{mmHg}^{*}$ & $100.84 \pm 11.2$ & $80 \pm 10.1$ & $<0.0001$ & $108 \pm 10.1$ & $77.19 \pm 7.36$ & $<0.0001$ \\
\hline FBG, $\mathrm{mmol} / \mathrm{L}$ & $4.78 \pm 0.63$ & $4.19 \pm 0.64$ & 0.02 & $5.17 \pm 1.35$ & $4.25 \pm 0.66$ & 0.021 \\
\hline $\mathrm{TC}, \mathrm{mmol} / \mathrm{L}$ & $4.22 \pm 0.92$ & $4.42 \pm 1.01$ & 0.5 & $4.07 \pm 0.95$ & $4.26 \pm 0.83$ & 0.19 \\
\hline $\mathrm{TG}, \mathrm{mmol} / \mathrm{L}$ & $1.62 \pm 0.84$ & $1.05 \pm 0.32$ & 0.01 & $1.32 \pm 0.49$ & $1.14 \pm 0.29$ & 0.03 \\
\hline HDL, mmol/L & $1.10 \pm 0.25$ & $1.18 \pm 0.23$ & 0.8 & $1.09 \pm 0.23$ & $1.03 \pm 0.16$ & 0.46 \\
\hline LDL, mmol/L & $2.46 \pm 0.70$ & $1.79 \pm 0.45$ & 0.12 & $2.17 \pm 0.86$ & $1.95 \pm 0.59$ & 0.04 \\
\hline
\end{tabular}

The data for age, Weight, SBP, DBP, FGB, TG, TC, HDL and LDL were presented as mean \pm SD. P-values for age, weight, SBP, DBP, FGB, HDL, LDL, TG, and TC were calculated using Student's t-test, $(\mathrm{P}<0.05)$. SBP, systolic blood pressure; $\mathrm{DBP}$, diastolic blood pressure; FBG, fasting blood glucose; HDL, high density lipoprotein; LDL, low density lipoprotein; TG, triglycerides; and TC, total cholesterol. *Grade 2: current blood pressure 160/100 179/109; Grade 3: current blood pressure $\geq 180 / 110$.

Table 2: Characteristics of study participants. 
Citation: Mushtaq N, Hussain S, Yuan L, Zhang S, Li H, et al. (2018) Gender Based Compositional Fluctuations in Gut Microbiota in Hypertension. Cardiovasc Pharm Open Access 7: 248. doi: 10.4172/2329-6607.1000248

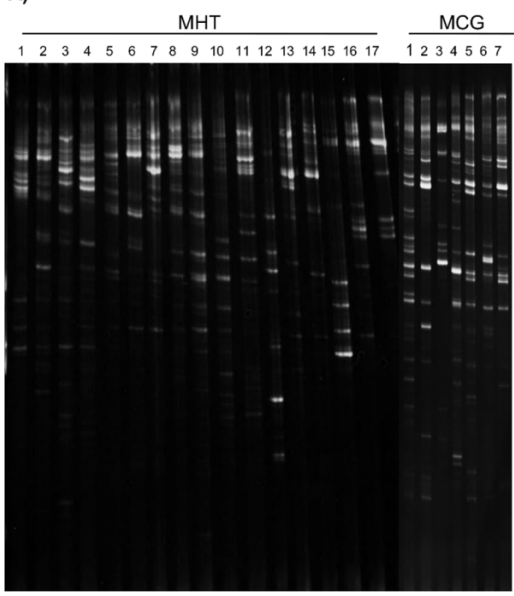

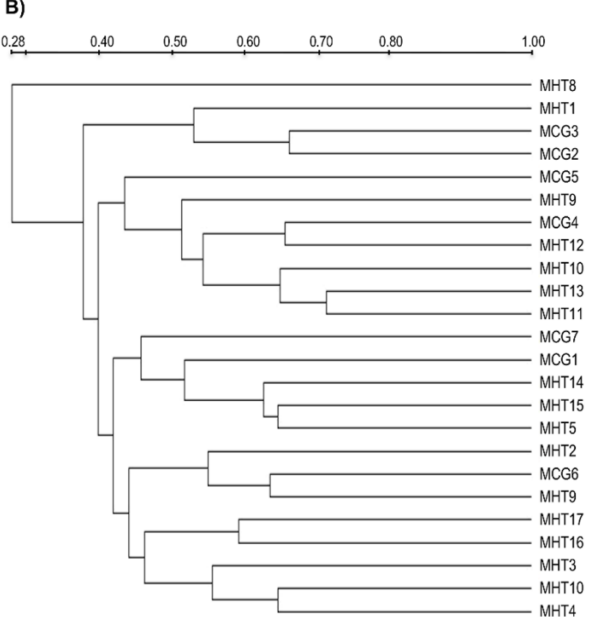

Figure 1: (A) DGGE profiles constructed by linkage primer with V3 region of $16 \mathrm{~S}$ rRNA gene of MHT and MCG groups. (B) Cluster analysis of MHT and MCG by applying UPGMA and Dice coefficient.

A)

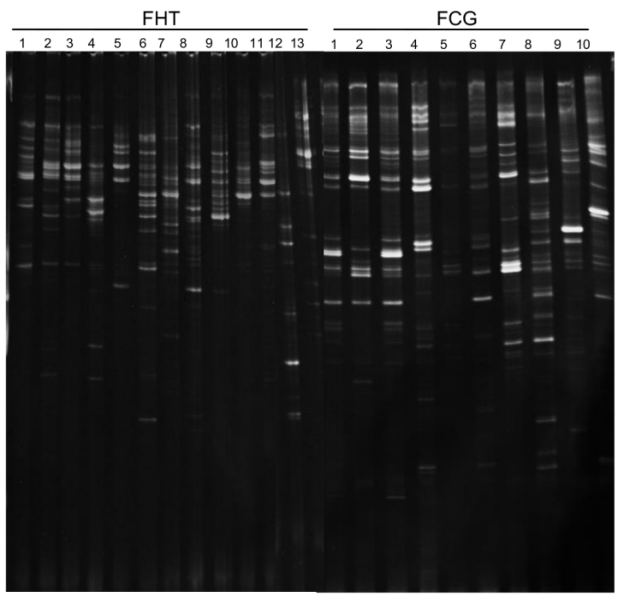

B)

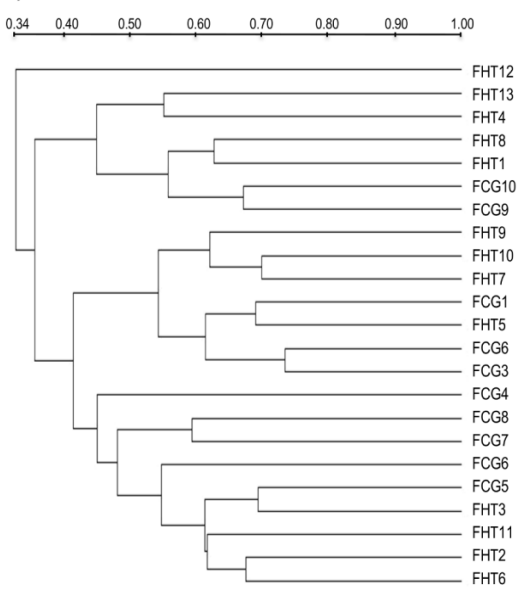

Figure 2: (A) DGGE profile constructed by linkage primers with V3 region of $16 \mathrm{~S}$ rRNA gene of FHT and FCG groups. (B) Cluster analysis of FHT and FCG by applying UPGMA and Dice coefficient.

\begin{tabular}{|c|c|c|c|}
\hline \multirow{2}{*}{ Group } & \multicolumn{2}{|c|}{ Microbiota diversity } & \multicolumn{2}{|c|}{ Microbiota similarity } \\
\cline { 2 - 4 } & $\begin{array}{c}\text { SGGE bands } \\
(\text { mean } \pm \text { SD })\end{array}$ & $\begin{array}{c}\text { Shannon index } \\
\text { (mean H'/H' max } \pm \text { SD) }\end{array}$ & Intra-group $^{\mathrm{c}}$ \\
\hline FHT & $16.23 \pm 3.71$ & $2.66 \pm 0.25$ & $44.78 \pm 12.46$ \\
\hline FCG & $19.8 \pm 5.20$ & $2.97 \pm 0.29$ & $41.78 \pm 12.91$ \\
\hline MHT & $15.64 \pm 4.74$ & $2.91 \pm 0.30$ & $42.69 \pm 11.29$ \\
\hline MCG & $13.14 \pm 5.39$ & $2.67 \pm 0.07$ & $40.32 \pm 8.52$ \\
\hline$P$ value FHT-FCG & 0.06 & 0.64 & $/$ \\
\hline
\end{tabular}

Values are presented as means \pm SD. Data were analyzed using the Student's $t$-test. aNumber of DGGE bands produced by each sample analyzed. 'Shannon Diversity Index as calculated using the relative intensities of all DGGE bands in each sample and expressed as a ratio of $\mathrm{H}^{\prime}$ to $\mathrm{H}^{\prime}$, where $\mathrm{H}^{\prime}$, is the maximum value of the Shannon index for a given sample. 'Dice similarity coefficients comparing DGGE band profiles within individuals of the same group. ${ }^{\mathrm{d} D i c e}$ similarity coefficients comparing DGGE band profiles between members of the study groups.

Table 3: Microbiota diversity and similarity of hypertensive patient groups compared with control groups. 
increased in the female hypertensive patients as compared to the healthy counterparts. No other significant differences were observed among the study groups. The above generated data is summarized in Table 4 . The triplicate samples were used to calculate the mean value in each experiment.

\section{High-throughput sequencing analysis of gene sequence}

The comparative statistics of the PCR sequences were estimated with 1,523,644 amplicons at the V3-V4 site of 16S rRNA gene from 20 hypertensive patients ( 10 females and 10 males) and 10 healthy controls (5 females and 5 males). Among these, sum of high-throughput sequencing reads $1,395,074$ with an average of $46,502.47$ per sample were passed for quality control and processed for further analysis. Taxon tag was $36,249.26$ (average) in all samples. The total number of OTUs assigned was 5786 with an average of 193 per sample in our study. The sum of the unique tags from the four groups was 307593 with an Avg. of 10,253.1 for all samples; that revealed the total phylotypes in this study. After deletion of linkage primers, the length of the average sequence was $422 \mathrm{bp}$.

\section{Gut microbial diversity and composition}

The total gut microbiota was evaluated for richness and diversity, as computed at $97 \%$ resemblance level. Rarefaction curves analysis indicated that the microbial communities were well represented for most of the samples and there were no obvious differences in the rarefaction curves of the four groups under study (Figure 3). Alpha diversity, as measured by Shannon index was significantly higher in the female control group than those in the female hypertensive group $(P=0.03)$, while the Simpson diversity in the male hypertensive patients was significantly higher as compared to the male control group $(P=0.01)$. Good's coverage was significantly decreased in the female hypertensive group while increased in the male hypertensive group as compared to their control groups (female and male, respectively). However, we could not observe the statistically significant differences in ACE and Chao 1 algorithms (Table 5).

\section{The relative abundance of gut microbiota in study groups}

The bacterial composition was assessed on the taxonomic basis at six levels and the taxa that composed of more than 1-0.5\% were considered. The total usable sequences were classified into 15 phyla, 32 classes, 51 orders, 78 families, 174 genera and 110 species. Among the 15 phyla sequenced, composition of gut microbiota was changed in-between the study groups. The majority of the obtained OTUs belonged to four phyla (female vs. male): Bacteroidetes (58\% vs. 64.2\%), Firmicutes (31.9\% vs. $28.8 \%)$, Proteobacteria ( $8.7 \%$ vs. $4.2 \%)$ and Actinobacteria ( $2.1 \%$ vs. $2.1 \%)$. The relative abundance of Firmicutes was significantly increased in the fecal microbiota of male and female hypertensive patients as compared to their control groups. The relative abundance of the phylum Bacteroidetes was decreased in the both hypertensive groups but the only statistically significant quantitative difference was observed between male hypertensive patients and male control group $(P=0.03)$ (Figure 4). The statistical data of the top 10 phyla is depicted in Table S2.

\section{Gut microbiota population at family level}

\begin{tabular}{|c|c|c|c|c|c|}
\hline \multirow{2}{*}{ Bacteria } & \multicolumn{2}{|c|}{ Female } & & \multicolumn{2}{|c|}{ Male } \\
\cline { 2 - 5 } & HTN & CG & $P$ value & HTN \\
\hline Bacteroides & $6.69 \pm 0.98$ & $7.14 \pm 0.48$ & 0.09 & $5.86 \pm 1.75$ & $6.78 \pm 1.45$ \\
\hline Prevotella & $4.82 \pm 1.84$ & $3.43 \pm 1.01$ & 0.03 & $5.84 \pm 2.13$ & $5.73 \pm 1.85$ \\
\hline Clostridium & $5.71 \pm 0.71$ & $5.54 \pm 0.39$ & 0.07 & $5.97 \pm 0.67$ & $5.37 \pm 0.39$ \\
\hline Escherichia coli & $4.39 \pm 1.24$ & $3.71 \pm 0.76$ & 0.19 & $4.38 \pm 1.04$ & 0.04 \\
\hline
\end{tabular}

Data were reported as the average estimate of Logarithms of fecal PCR target genetic amplicon copy numbers present in $1 \mathrm{~g}$ of feces. Results which are significantly different (Student's $t$-test), with $P<0.05$.

Table 4: Quantitative analysis of bacterial groups in fecal samples by real-time PCR (qPCR).
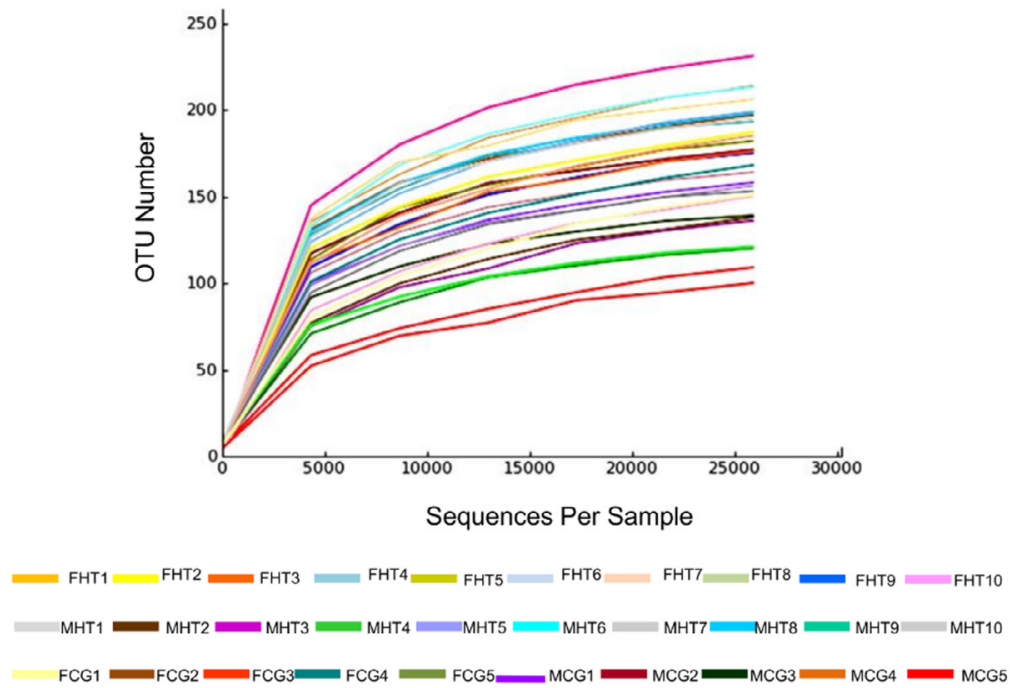

Figure 3: Rarefaction curves used to estimate richness among the study groups at $97 \%$ similarity level. 
Among the 10 top most families, the prevalence of Prevotellaceae, Veillonellaceae, Peptostreptococcaceae were higher in the female hypertensive patients. On the other hand, Bacteroidaceae and Ruminococcaceae were significantly decreased and increased respectively, in the male hypertensive patients (Figure 5). The family level data statistics which illustrates the quantitative difference are displayed in Table S2.

\section{Gut microbiota distribution at genus level}

Among the 10 top most genera, a significantly increased abundance of Prevotella_9 $(P=0.012)$ and Megasphaera $(P=0.017)$ was observed in female hypertensive patients, while significant increase and decrease of Megamonas ( $P=0.013$ ) and Bacteroides $(P=0.013)$ respectively, were observed in male hypertensive patients as compared to their control group (Figure 6). The dominant phylotypes in female and male hypertensive patients were also different. The quantitative differences at genera level between the study groups are compiled in Table S2.

\section{Gut microbial distribution at species level}

A total of 110 sequences were classified at the specie level and among these, some of the species revealed significant differences in their relative abundance in the hypertensive and control groups (Table S3).

\section{Correlation of molecular protocols}

The data obtained from this study using the molecular techniques

\begin{tabular}{|c|c|c|c|c|c|c|}
\hline Group & $\begin{array}{c}\text { Observed } \\
\text { species }\end{array}$ & Shannon & Simpson & Chao1 & ACE & Good \\
\hline FHT & 170.4 & 3.90 & 0.86 & 193.90 & 197.12 & 0.9987 \\
\hline FCG & 165.5 & 4.45 & 0.91 & 185.37 & 183.78 & 0.999 \\
\hline MHT & 183.5 & 4.20 & 0.96 & 200.43 & 205.48 & 1.0899 \\
\hline MCG & 172.5 & 4.12 & 0.86 & 197.63 & 202.44 & 0.9986 \\
\hline \multicolumn{7}{|c|}{$\boldsymbol{P}$ values } \\
\hline FHT-FCG & 0.23 & 0.03 & 0.07 & 0.95 & 0.73 & 0.01 \\
\hline MHT-MCG & 0.58 & 0.11 & 0.01 & 0.58 & 0.57 & 0.001 \\
\hline
\end{tabular}

The number shown in this table is the mean value of each group. Results which are significantly different (Student's $t$-test), with $P<0.05$.

Table 5: Gut bacterial richness and diversity index that were based on $97 \%$ similarity by high-throughput sequencing analysis.

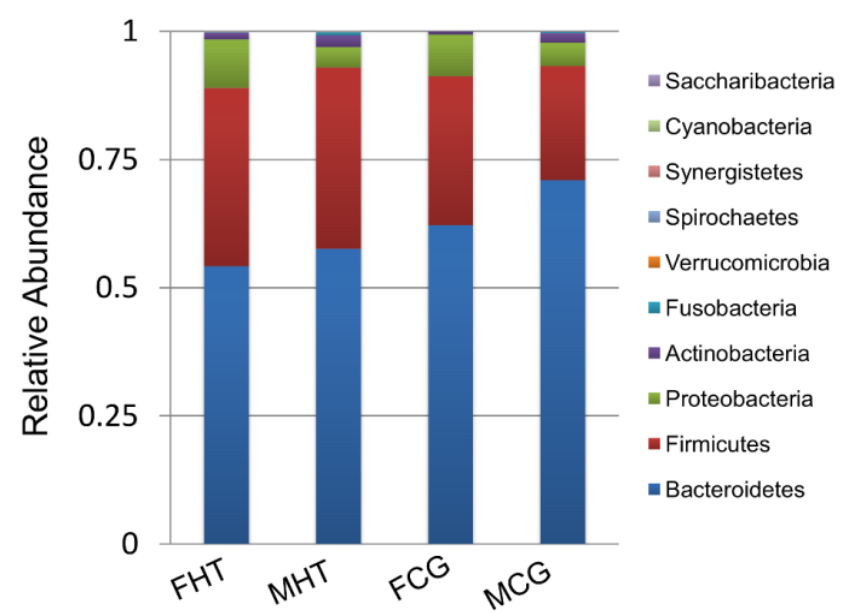

Figure 4: Gut microbial composition at phylum level obtained from highthroughput sequencing results.

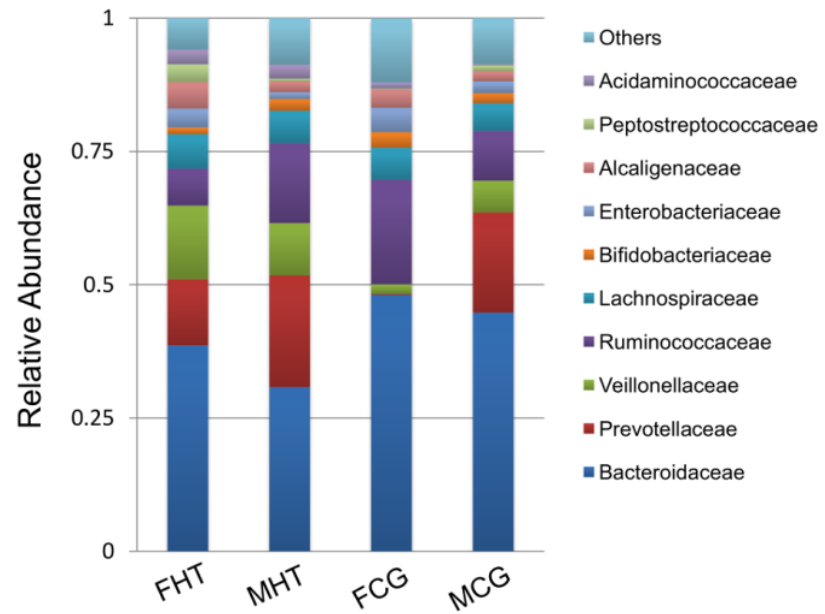

Figure 5: Gut microbial composition at family level obtained from highthroughput sequencing results.

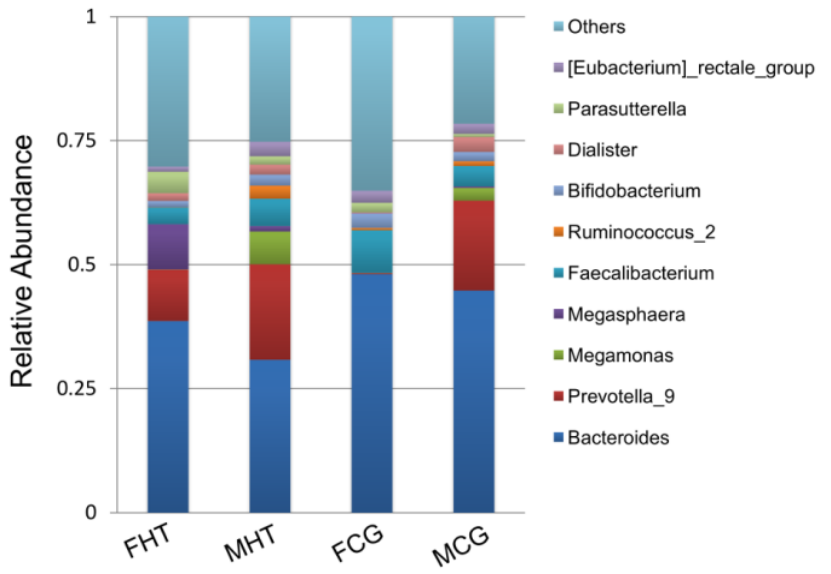

Figure 6: The relative abundance of 10 highly prevalent genera in the study groups obtained from High-throughput sequencing analysis.

including DGGE and High-throughput sequencing revealed the most prevalent bacterial phyla in the gut i.e. Bacteroidetes, Firmicutes, Proteobacteria and Actinobacteria. The PCR-DGGE analysis is economical and less time consuming, and is considered a basic technique to scrutinize the substantial shift of gut bacterial populations. On the other hand, High-throughput Illumina sequencing is much reliable, sensitive and authentic technique to validate the diverse bacterial communities. The findings of the PCR-DGGE analysis were aligned with the High-throughput sequencing results.

\section{Discussion}

Human gut microbiota plays critical role in body defense in response to various anomalies mediated by metabolic malfunctions and pathologies caused by multiple diseases. The experimental data from current study establish the substantial alterations between gut microbial populations of male and female hypertensive patients and healthy subjects, as evident by DGGE profiling, High-throughput sequencing and Real-time PCR analysis. The $\alpha$ - diversity calculated by Shannon and Simpson indexes was significantly decreased and increased in female hypertensive and male hypertensive patients 
respectively, as compared to their healthy controls. Good's coverage was also significantly decreased and increased in female and male hypertensive patients, respectively. Conversely no significant differences were found by statistical analysis of nonparametric Chao 1, algorithm ACE and observed species. Moreover, the bacterial population diversity as estimated by the DGGE profiling and validated by High-throughput sequencing was lower in female patients but higher in male patients. We determined the gut microbiota distribution at the phyla, family, genera and specie levels and observed the variations among the study groups.

The relative abundance of Firmicutes and Bacteroidetes wasincreased and decreased in female and male hypertensive patients respectively, as compared to their healthy. Moreover, the most differentially abundant bacterial taxa belonged to the genera Prevotella and Megasphaera in female hypertensive patients and Megamonas in male hypertensive patients. Further, the female and male hypertensive patients also showed different dominant phylotypes comparing in between them and to their healthy counterparts. These results demonstrate that there are sex based differences in the gut microbial composition of individuals with hypertension, and categorize Prevotella, Megasphaera and Megamonas as the sex-specific bacteria associated with hypertension. Recently we have found that the relative abundance of Bacteroidetes and Firmicutes is significantly decreased and increased, respectively, in hypertensive patients as compared to the healthy controls (data not published yet). In our last study we investigated the gut microbiota dysbiosis and its causal role in the development of hypertension, and the sex-based evaluation was not taken into consideration. However, sex-based differences are prominent in hypertension as reported by earlier studies $[8,9]$. Analyzing the differential gut microbiota in female and male hypertensive patients and their healthy counterparts, we found that the Bacteroidetes were decreased significantly only in the male hypertensive patients while Firmicutes were increased significantly, in male and female (both) hypertensive groups.

Clinically, hypertension and metabolic-diseases such as diabetes and obesity are considered as interrelated, associated with common etiological factors [15]. Previous studies have shown that low Bacteroidetes levels were associated with obesity [40]. Therefore, we excluded the obese and diabetic subjects to rule out the potential influence of these factors in our current study. In addition, the BMI difference between hypertensive patients and healthy controls was almost negligible. So, the obesity or diabetes could not be the confounding factors in this study. Although no significant variability in the overall abundance of Bacteroidetes between female hypertensive patients and female healthy controls were identified, some Bacteroidetes OTUs in female patients were increased, while others were decreased. Therefore, the fluctuated Bacteroidetes could still be a hallmark in female hypertensive patients. On the other hand, male patients group showed significant decrease in the relative abundance of Bacteroides as compared to the male control group. Bacteroides are the beneficial bacteria and maintain a complex relationship with host in the gut, acting as commensals [41]. These bacteria are known to cause the fermentation of a wide range of sugar derivatives from plant materials (which are common in the human colon and are potentially toxic) making them beneficial for humans. Bacteroides and other intestinal bacteria cause carbohydrates fermentation and produce volatile fatty acids that are reabsorbed through the large intestine and utilized by the host as an energy source, meeting the considerable proportion of our daily energy requirement [42].

We observed significantly raised levels of Prevotella in female hypertensive patients. Prevotella plays a central role in digesting plant food. It has also been reported that Prevotella participates in urinary tract infections, arthritis, osteomyelitis and brain abcessess [43,44]. Ruminococcus spp., Faecalibacterium spp., and Roseburia spp. are significantly decreased in inflammatory bowel disease (IBD) and acute colitis, suggesting that these bacteria are imperative to maintain the intestinal homeostasis [45]. Faecalibacterium prausnitzii is an antiinflammatory commensal bacterium and was found significantly reduced in patients with active IBD rather than those in remission [46] and patients with recurrent IBD [47]. Additionally, F. prausnitzii was reported to stimulate peripheral blood mononuclear cells (PBMCs) for the secretion of anti-inflammatory cytokine IL-10, whereas it inhibited the production of pro-inflammatory cytokines, such as IL-12 and IFN- $\gamma$ [47]. Our data showed a significant reduction of Ruminococcus and Roseburia in female hypertensive patients only. On the other hand, F. prausnitzii was significantly decreased in both male and female hypertensive patients, suggesting the significant role of these bacteria in sex related hypertension.

Phascolarctobacterium spp. and Veillonellaceae, both produce high amounts of short chain fatty acids, acetate and propionate, and belong to the same class within the phylum Firmicutes $[48,49]$. The butyrate is mainly used by colonocytes, while acetate and propionate are largely taken up by the liver. Acetate promotes hypercholesterolemia, hypertriglyceridemia and results in liver steatosis as it required for cholesterol and fatty acid production. Propionate inhibits cholesterol synthesis in liver tissue and acts as a substrate for hepatic gluconeogenesis. Propionate is also reported to play a role in decreasing the plasma lipid levels [50] however, its anti-lipogenesis effects are divisive. Besides, propionate promotes inhibition of lipolysis and adipocyte differentiation, mediated by the activation of $G$ proteincoupled receptor 43 or free fatty acid receptor 2, causing increased adiposity $[51,52]$. The members of the Veillonellaceae family are usually responsible for polymicrobial and rarely for monomicrobial infections in humans such as osteoarticular infections or endocarditis [53]. In the present study we observed an increased abundance of family Veillonellaceae in male and female hypertensive patients as compared to the normal male and female groups.

Many of the taxonomies identified in the current study however, are novel and are reported for the first time. The genus Bacteroides and family Enterobacteriaceae are both negatively associated with triglycerides and play role in bile acid metabolism [11]. Bile acid activity of commensal bacteria is involved in a complex interplay with host hepatic enzymes, and together they promote digestion and absorption of dietary lipids [54]. Triglycerides levels were found raised in the participants of both male and female hypertensive patients groups of our study, which indicates the role of bacterial bile acids in lipid metabolism. Diet is considered an important factor influencing gut microbiota community. In the present study we could not evaluate the relationship of gut microbiota with dietary habits of the study groups because of the incomplete nutritional information. However, our conclusions are unlikely to be affected by this potential confounding factor, due to almost similar dietary habits and lifestyle of the enrolled subjects from Xian city of China.

The Firmicutes and Bacteroidetes (F/B) ratio has been reported to be increased in hypertension, and the oral administration of minocycline has been found to normalize the $\mathrm{F} / \mathrm{B}$ ratio and blood pressure in hypertension [17]. Further, manipulating gut microbiota using pre- and probiotics might prove a valuable adjuvant to traditional antihypertensives. Pre- and probiotics modulate cholesterol, inflammation, blood glucose levels, and the renin-angiotensin system to lower BP and risk for HTN [55]. A meta-analysis suggested that the continuous use of probiotics for $\geq 8$ weeks may lower BP by a modest 
degree, with potentially greater effect when BP is elevated [56-59]. However, a detailed understanding of gut microbial composition in relation to $\mathrm{BP}$, ethnicity etc must be evaluated to fully recognize the implications of pre- and probiotics in HTN therapeutics.

\section{Conclusion}

This study elaborates the gender based compositional differences in the gut microbiota and identifies sex-specific bacterial populations in human hypertensive patients, comparing with their healthy counterparts. The most distinctive and differentially abundant bacterial taxa belonged to Prevotella and Megasphaera in female, while Megamonas in male hypertensive patients, respectively. These results provide novel insights for developing new strategies to manage hypertension and its related diseases. However, the suitability of Prevotella, Megasphaera and Megamonas may be further evaluated as the gender-specific biomarkers for diagnosing hypertension.

\section{Acknowledgments}

This project was funded by "National Natural Science Foundation of China (NSFC81730056)". We would like to thank the "Center for Disease Control and Prevention" of Shaanxi province for the equipment and technical support.

\section{Supplementary Material}

The data for sequencing of PCR amplicons from DGGE gel profile and recognition linking to BLAST database; Gut microbial phylotypes from Highthroughput sequencing; and the significantly divergent gut microbial species from High- throughput sequencing, is given in a separate file (Supplemental File) as Table S1; Table S2; and Table S3, respectively.

\section{References}

1. Pierdomenico SD, Di Nicola M, Esposito AL, Di Mascio R, Ballone E, et al. (2009) Prognostic value of different indices of blood pressure variability in hypertensive patients. Am J Hypertens 22: 842-847.

2. Hillege HL, Girbes AR, De Kam PJ, Boomsma F, De Zeeuw D, et al. (2000) Renal function, neurohormonal activation, and survival in patients with chronic heart failure. Circulation 102: 203-210.

3. Ong KL, Cheung BM, Man YB, Lau CP, Lam KS (2007) Prevalence, awareness, treatment, and control of hypertension among United States adults 1999-2004. Hypertension 49: 69-75.

4. Buttar HS, Li T, Ravi N (2005) Prevention of cardiovascular diseases: Role of exercise, dietary interventions, obesity and smoking cessation. Exp Clin Cardiol 10: 229-249.

5. Husain K, Ansari RA, Ferder L (2014) Alcohol-induced hypertension: Mechanism and prevention. World J Cardiol 6: 245-252.

6. Matsuda M, Shimomura I (2013) Increased oxidative stress in obesity: implications for metabolic syndrome, diabetes, hypertension, dyslipidemia, atherosclerosis, and cancer. Obes Res Clin Pract 7: e330-341.

7. Mathers CD, Loncar D (2006) Projections of global mortality and burden of disease from 2002 to 2030 . PLoS Med 3: e442.

8. Daugherty SL, Masoudi FA, Ellis JL, Ho PM, Schmittdiel JA, et al. (2011) Agedependent gender differences in hypertension management. J Hypertens 29: 1005-1011.

9. Chu SH, Baek JW, Kim ES, Stefani KM, Lee WJ, et al. (2015) Gender differences in hypertension control among older korean adults: Korean social life, health, and aging project. J Prev Med Public Health 48: 38-47.

10. Sandberg K, Ji H (2012) Sex differences in primary hypertension. Biol Sex Differ 3: 7 .

11. Vitale C, Fini M, Speziale G, Chierchia S (2010) Gender differences in the cardiovascular effects of sex hormones. Fundam Clin Pharmacol 24: 675-685.

12. Markle JG, Frank DN, Mortin-Toth S, Robertson CE, Feazel LM, et al. (2013) Sex differences in the gut microbiome drive hormone-dependent regulation of autoimmunity. Science 339: 1084-1088.

13. Bhupathy $P$, Haines CD, Leinwand LA (2010) Influence of sex hormones and phytoestrogens on heart disease in men and women. Womens Health 6: 77-95.
14. Luczak ED, Leinwand LA (2009) Sex-based cardiac physiology. Annu Rev Physiol 71: 1-18.

15. Tang WH, Wang Z, Levison BS, Koeth RA, Britt EB, et al. (2013) Intestinal microbial metabolism of phosphatidylcholine and cardiovascular risk. N Engl J Med 368: 1575-1584.

16. Durgan DJ, Ganesh BP, Cope JL, Ajami NJ, Phillips SC, et al. (2016) Role of the Gut Microbiome in Obstructive Sleep Apnea-Induced Hypertension. Hypertension 67: 469-474.

17. Yang T, Santisteban MM, Rodriguez V, Li E, Ahmari N, et al. (2015) Gut dysbiosis is linked to hypertension. Hypertension 65: 1331-1340.

18. Mell B, Jala VR, Mathew AV, Byun J, Waghulde H, et al. (2015) Evidence for a link between gut microbiota and hypertension in the Dahl rat. Physiol Genomics 47: 187-197.

19. Gkaliagkousi E, Passacquale G, Douma S, Zamboulis C, Ferro A (2010) Platelet activation in essential hypertension: implications for antiplatelet treatment. Am J Hypertens 23: 229-236.

20. Zhu W, Gregory JC, Org E, Buffa JA, Gupta N, et al. (2016) Gut Microbia Metabolite TMAO Enhances Platelet Hyperreactivity and Thrombosis Risk. Cell 165: $111-124$

21. Costello EK, Lauber CL, Hamady M, Fierer N, Gordon JI, et al. (2009) Bacterial community variation in human body habitats across space and time. Science 326: 1694-1697.

22. Org E, Parks BW, Joo JW, Emert B, Schwartzman W, et al. (2015) Genetic and environmental control of host-gut microbiota interactions. Genome Res 25 : 1558-1569.

23. Wu GD, Chen J, Hoffmann C, Bittinger K, Chen YY, et al. (2011) Linking longterm dietary patterns with gut microbial enterotypes. Science 334: 105-108.

24. David LA, Maurice CF, Carmody RN, Gootenberg DB, Button JE, et al. (2014) Diet rapidly and reproducibly alters the human gut microbiome. Nature 505: 559-563.

25. Van Nas A, Guhathakurta D, Wang SS, Yehya N, Horvath S, et al. (2009) Elucidating the role of gonadal hormones in sexually dimorphic gene coexpression networks. Endocrinology 150: 1235-1249.

26. Kovacs A, Ben-Jacob N, Tayem H, Halperin E, Iraqi FA, et al. (2011) Genotype is a stronger determinant than sex of the mouse gut microbiota. Microb Ecol 61: 423-428.

27. Lay C, Rigottier-Gois L, Holmstrom K, Rajilic M, Vaughan EE, et al. (2005) Colonic microbiota signatures across five northern European countries. Appl Environ Microbiol 71: 4153-4155.

28. Huttenhower C, Gevers D, Knight R, Abubucker S (2012) Structure Function and diversity of the healthy human microbiome. Nature 486: 207-214.

29. Qin J, Li R, Raes J, Arumugam M, Burgdorf KS, et al. (2010) A human gut microbial gene catalogue established by metagenomic sequencing. Nature 464: 59-65.

30. Mueller S, Saunier K, Hanisch C, Norin E, Alm L, et al. (2006) Differences in fecal microbiota in different European study populations in relation to age gender, and country: a cross-sectional study. Appl Environ Microbiol 72: $1027-$ 1033.

31. Shastri P, McCarville J, Kalmokoff M, Brooks SP, Green-Johnson JM (2015) Sex differences in gut fermentation and immune parameters in rats fed an oligofructose-supplemented diet. 6: 13.

32. Yurkovetskiy L, Burrows M, Khan AA, Graham L, Volchkov P, et al. (2013) Gender bias in autoimmunity is influenced by microbiota. Immunity 39: 400412.

33. Yuan L, Zhang S, Li H, Yang F, Mushtaq N, et al. (2018) The influence of gut microbiota dysbiosis to the efficacy of 5-Fluorouracil treatment on colorectal cancer. Biomed Pharmacother 108: 184-193.

34. Muyzer G, De Waal EC, Uitterlinden AG (1993) Profiling of complex microbia populations by denaturing gradient gel electrophoresis analysis of polymerase chain reaction-amplified genes coding for $16 \mathrm{~S}$ rRNA. Appl Environ Microbiol 59: 695-700.

35. Peiffer JA, Spor A, Koren O, Jin Z, Tringe SG, et al. (2013) Diversity and heritability of the maize rhizosphere microbiome under field conditions. Proc Natl Acad Sci USA 110: 6548-6553. 
Citation: Mushtaq N, Hussain S, Yuan L, Zhang S, Li H, et al. (2018) Gender Based Compositional Fluctuations in Gut Microbiota in Hypertension. Cardiovasc Pharm Open Access 7: 248. doi: 10.4172/2329-6607.1000248

Page 9 of 9

36. Caporaso JG, Kuczynski J, Stombaugh J, Bittinger K, Bushman FD, et al. (2010) QIIME allows analysis of high-throughput community sequencing data. Nat Methods 7: 335-336.

37. Magoc T, Salzberg SL (2011) FLASH: fast length adjustment of short reads to improve genome assemblies. Bioinformatics 27: 2957-2963.

38. Edgar RC (2010) Search and clustering orders of magnitude faster than BLAST. Bioinformatics 26: 2460-2461.

39. Wang Q, Garrity GM, Tiedje JM, Cole JR (2007) Naive Bayesian classifier for rapid assignment of rRNA sequences into the new bacterial taxonomy. Appl Environ Microbiol 73: 5261-5267.

40. Everard A, Cani PD (2013) Diabetes, obesity and gut microbiota. Best Pract Res Clin Gastroenterol 27: 73-83.

41. Xu J, Gordon JI (2003) Honor thy symbionts. Proc Natl Acad Sci USA 100 10452-10459.

42. Hooper LV, Midtvedt T, Gordon JI (2002) How host-microbial interactions shape the nutrient environment of the mammalian intestine. Annu Rev Nutr 22: $283-307$

43. Scher JU, Sczesnak A, Longman RS, Segata N, Ubeda C, et al. (2013) Expansion of intestinal Prevotella copri correlates with enhanced susceptibility to arthritis. Elife 2: e01202.

44. Dillon SM, Lee EJ, Kotter CV, Austin GL, Gianella S, et al. (2016) Gut dendritic cell activation links an altered colonic microbiome to mucosal and systemic T-cell activation in untreated HIV-1 infection. Mucosal Immunol 9: 24-37.

45. Rao PM, Kelly DM, Jones TH (2013) Testosterone and insulin resistance in the metabolic syndrome and T2DM in men. Nat Rev Endocrinol 9: 479-493.

46. Burt VL, Whelton P, Roccella EJ, Brown C, Cutler JA, et al. (1995) Prevalence of hypertension in the US adult population. Results from the Third National Health and Nutrition Examination Survey, 1988-1991. Hypertension 25: 305313.

47. Sokol H, Pigneur B, Watterlot L, Lakhdari O, Bermudez-Humaran LG, et al. (2008) Faecalibacterium prausnitzii is an anti-inflammatory commensal bacterium identified by gut microbiota analysis of Crohn disease patients. Proc Natl Acad Sci USA 105: 16731-16736.

48. Duncan SH, Holtrop G, Lobley GE, Calder AG, Stewart CS, et al. (2004) Contribution of acetate to butyrate formation by human faecal bacteria. $\mathrm{Br} J$
Nutr $91: 915-923$

49. WatanabeY,Nagai F, MorotomiM(2012)Characterization of Phascolarctobacterium succinatutens sp. nov., an asaccharolytic, succinate-utilizing bacterium isolated from human feces. Appl Environ Microbiol 78: 511-518.

50. Wong JM, De Souza R, Kendall CW, Emam A, Jenkins DJ (2006) Colonic health: fermentation and short chain fatty acids. J Clin Gastroenterol 40: 235243.

51. Ge H, Li X, Weiszmann J, Wang P, Baribault H, et al. (2008) Activation of $\mathrm{G}$ protein-coupled receptor 43 in adipocytes leads to inhibition of lipolysis and suppression of plasma free fatty acids. Endocrinology 149: 4519-4526.

52. Bjursell M, Admyre T, Goransson M, Marley AE, Smith DM, et al. (2011) Improved glucose control and reduced body fat mass in free fatty acid recepto 2-deficient mice fed a high-fat diet. Am J Physiol Endocrinol Metab 300: E211220 .

53. Marchandin H, Jumas-Bilak E (2014) The Family Veillonellaceae, in The Prokaryotes: Firmicutes and Tenericutes. Springer Berlin Heidelberg: Berlin, Heidelberg. PP: 433-453.

54. Ridlon JM, Kang DJ, Hylemon PB (2006) Bile salt biotransformations by human intestinal bacteria. J Lipid Res 47: 241-259.

55. Upadrasta A, Madempudi RS (2016) Probiotics and blood pressure: current insights. Integr Blood Press Control 9: 33-42.

56. Khalesi S, Sun J, Buys N, Jayasinghe R (2014) Effect of probiotics on blood pressure: a systematic review and meta-analysis of randomized, controlled trials. Hypertension 64: 897-903.

57. Huijsdens XW, Linskens RK, Mak M, Meuwissen SG, Vandenbroucke-Grauls CM, et al. (2002) Quantification of bacteria adherent to gastrointestinal mucosa by real-time PCR. J Clin Microbiol 40: 4423-4427.

58. Matsuki T, Watanabe K, Fujimoto J, Miyamoto Y, Takada T, et al. (2002) Development of $16 \mathrm{~S}$ rRNA-gene-targeted group-specific primers for the detection and identification of predominant bacteria in human feces. App Environ Microbiol 68: 5445-5451.

59. Bartosch S, Fite A, Macfarlane GT, McMurdo ME (2004) Characterization of bacterial communities in feces from healthy elderly volunteers and hospitalized elderly patients by using real-time PCR and effects of antibiotic treatment on the fecal microbiota. Appl Environ Microbiol 70: 3575-3581. 